\title{
Femoral Access and Delivery of Continuous Renal Replacement Therapy Dose
}

\author{
Rinaldo Bellomo $^{a, b}$ Johan Mårtensson ${ }^{b, c}$ Serigne Lo ${ }^{d}$ Kirsi-Maija Kaukonen ${ }^{a}$ \\ Alan Cass $^{\mathrm{e}}$ Martin Gallagher ${ }^{d}$ for the RENAL study investigators and the \\ Australian and New Zealand Intensive Care Clinical Trials Group \\ ${ }^{a}$ Australian and New Zealand Intensive Care Research Centre (ANZIC-RC), Department of Epidemiology and Preventive

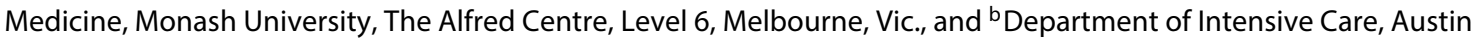 \\ Hospital, Heidelberg, Vic., Australia; 'Section of Anaesthesia and Intensive Care Medicine, Department of Physiology and \\ Pharmacology, Karolinska Institutet, Solna, Sweden; ${ }^{\mathrm{d}}$ The George Institute for Global Health, University of Sydney, Level \\ 13, Sydney, N.S.W., and ' Menzies School of Health Research, Charles Darwin University, Darwin, Winnellie, N.T., Australia
}

\section{Key Words}

Dialysis - Continuous renal replacement therapy ·

Catheters · Femoral vein · Intensive care

\begin{abstract}
Aims: The study aims to describe the use of dialysis catheters in critically ill patients treated with continuous renal replacement therapy (CRRT) and to study the impact of femoral versus non-femoral access on CRRT dose. Methods: Statistical analysis and predictive modelling of data from the Randomized Evaluation of Normal vs. Augmented Level renal replacement therapy trial. Results: The femoral vein was the first access site in 937 (67\%) of 1,399 patients. These patients had higher Acute Physiology and Chronic Health Evaluation and Sequential Organ Failure Assessment scores $(p=$ $0.009)$ and lower $\mathrm{pH}(\mathrm{p}<0.001)$ but similar mortality to patients with non-femoral access ( 44 vs. $45 \% ; p=0.63$ ). Lower body weight was independently associated with femoral access placement (OR $0.97,95 \% \mathrm{Cl} 0.96-0.98)$. Femoral access was associated with a $1.03 \%$ lower CRRT dose $(p=0.05)$, but a $4.20 \%$ higher dose was achieved with $13.5 \operatorname{Fr}$ catheters $(p=$
\end{abstract}

0.03). Conclusions: Femoral access was preferred in lighter and sicker patients. Catheter gauge had greater impact than catheter site in CRRT dose delivery.

Video Journal Club "Cappuccino with Claudio Ronco" at http://www.karger.com/?doi=439581. @ 2015 s. Karger AG, Basel

\section{Introduction}

The ability to maintain patient homeostasis of water, waste products, electrolytes and acid-base during continuous renal replacement therapy (CRRT) is dependent on CRRT circuit patency over time [1]. The quality of vascular access influences the ability to achieve adequate and reliable blood flow through the circuit, a major determinant of circuit life span. Catheter dysfunction requiring

A complete list of investigators in the RENAL study is provided in the supplementary material (online suppl. item S1; for all online suppl. material, see www.karger.com/doi/10.1159/000439581).

\section{KARGER}

E-Mail karger@karger.com

www.karger.com/bpu (c) 2015 S. Karger AG, Basel

0253-5068/15/0413-0011\$39.50/0
Prof. Rinaldo Bellomo

Department of Intensive Care, Austin Health

145 Studley Road

Heidelberg, VIC 3084 (Australia)

E-Mail rinaldo.bellomo@ austin.org.au 
catheter replacement and/or causing circuit failure occurs in approximately $10 \%$ of patients treated with CRRT $[2,3]$. Such circuit failures induce interruptions in treatment, which decrease the delivered CRRT dose and uremic control [4]. Moreover, they significantly increase nursing workload and treatment costs [5]. Thus, both choice of optimal insertion site and catheter characteristics are important to delivering optimal therapy.

There is uncertainty regarding the optimal site of catheter insertion for CRRT in critically ill patients. Femoral vein catheterization may be faster and easier than jugular vein catheterization [6] but may impede mobilization [7]. Thus, recent guidelines favor the right jugular vein in preference to femoral veins [8]. These recommendations are, however, not supported by robust evidence. Furthermore, the impact of catheter brand, gauge and length on the delivery of CRRT has only been investigated in small studies and no robust evidence exists to guide choice of catheter.

The Randomized Evaluation of Normal vs. Augmented Level of Replacement Therapy (RENAL) study collected detailed information on dialysis catheter characteristics, site of catheter insertion and circuit anticoagulation during CRRT and therefore offers a unique opportunity to explore the relationship between these factors and delivered CRRT dose [9-11]. Accordingly, we aimed to describe the use of temporary dialysis catheters in intensive care unit (ICU) patients treated with CRRT. In particular, we sought to identify factors associated with choosing the femoral vein as first site of catheter insertion and to explore the association of first insertion site (femoral vs. non-femoral) and different catheter characteristics with delivered CRRT dose during the first full day of treatment.

\section{Materials and Methods}

\section{Study Protocol}

The RENAL study was a prospective, multicenter, randomized, controlled trial comparing a higher $(40 \mathrm{ml} / \mathrm{kg} / \mathrm{h})$ versus a lower $(25 \mathrm{ml} / \mathrm{kg} / \mathrm{h})$ CRRT dose in 1,508 critically ill patients. The Human Research Ethics Committees of the University of Sydney and of all participating institutions approved the study. Written informed consent was obtained from patients or next of kin. Adult patients ( $\geq 18$ years) with severe AKI requiring acute RRT were included if they met at least one of the following criteria: oliguria (urine output $<100 \mathrm{ml}$ during $\geq 6 \mathrm{~h}$ not responding to fluid resuscitation), hyperkalemia (serum potassium $>6.5 \mathrm{mmol} / \mathrm{l}$ ), severe acidosis $(\mathrm{pH}$ $<7.2$ ), a serum urea level $>25 \mathrm{mmol} / \mathrm{l}$, a serum creatinine level $>300$ $\mu \mathrm{mol} / \mathrm{l}$ or the presence of clinically significant organ edema. $\mathrm{Pa}-$ tients with previous RRT during the same hospitalization or those with end-stage renal failure requiring chronic dialysis were excluded. A detailed study protocol is found in the appendix of the orig- inal study [11]. The primary end point was 90 -day mortality. Secondary end points included RRT-free days, mechanical ventilation-free days, ICU-free days and hospital-free days at 90-day follow-up.

The site of dialysis catheter insertion, catheter choice and mode of circuit anticoagulation were determined by the treating physician. For the purpose of the present study, the delivered CRRT dose (as a percentage of prescribed dose) during the first complete 24-hour day was recorded.

\section{Statistical Analysis}

Data was analyzed by using SAS software version 9.1. Continuous variables were expressed as mean (SD) and categorical variables as numbers (\%). The Student $t$ test or the Mann-Whitney test was used to compare continuous variables. The $\chi^{2}$ test or the Fisher's exact test was used to compare categorical variables. Multivariate logistic regression analysis was used to study the association of having the femoral vein as first site of catheter insertion. Baseline variables were considered and were included in the multivariate model if they were statistically significant at $p$ value $<0.20$ in the univariate analysis. The association between using a femoral catheter (vs. a non-femoral catheter) and CRRT dose during the first complete $24 \mathrm{~h}$ was assessed by multivariate linear regression analysis. The following potential confounders were considered: treatment group, baseline characteristics, illness severity, catheter gauge, catheter length, catheter brand, CRRT machine and anticoagulation mode. Covariates were included in the multivariate model if they were statistically significant at $p$ value $<0.20$ in the univariate analysis. In the final analyses, a 2 -sided $\mathrm{p}$ value $<0.05$ was considered statistically significant.

\section{Results}

\section{First Site of Catheter Insertion}

Of the 1,508 randomized patients in the RENAL trial, data on first dialysis catheter insertion site was available for 1,399 (93\%) patients. In 937 (67\%) of these 1,399 patients the femoral vein was chosen as the first site of dialysis catheter insertion (fig. 1). The right femoral vein was preferred over the left. Jugular access was used in 351 (25\%) patients and subclavian access was chosen in 111 (8\%) patients.

\section{Factors Associated with Femoral Vein Catheterization}

Demographics, admission diagnosis, time from ICU admission to randomization, treatment group, baseline biochemistry and outcomes for patients with femoral and non-femoral vein as first site of dialysis catheter insertion are detailed in table 1. Patients receiving femoral access weighed on an average $3.8 \mathrm{~kg}$ less than patients receiving a non-femoral access ( $\mathrm{p}<0.001$; table 1$)$ and had higher Acute Physiology and Chronic Health Evaluation (APACHE) III scores, cardiovascular Sequential Organ Failure Assessment scores, hemoglobin levels and chlo- 
Table 1. Characteristics of patients according to first site of catheter insertion

\begin{tabular}{|c|c|c|c|}
\hline Characteristic & $\begin{array}{l}\text { Femoral } \\
(n=937)\end{array}$ & $\begin{array}{l}\text { Non-femoral } \\
(\mathrm{n}=462)\end{array}$ & $\mathrm{p}$ values \\
\hline Age, years & $64.3 \pm 15.2$ & $65.0 \pm 14.2$ & 0.5 \\
\hline APACHE III score & $103.5(25.7)$ & $100.1(25.4)$ & 0.02 \\
\hline Time from ICU admission to randomization, $\mathrm{h}$ & $46.1 \pm 105$ & $62.5 \pm 145$ & 0.02 \\
\hline Randomized to high CRRT intensity & $456(48.7)$ & $236(51.1)$ & 0.40 \\
\hline Body weight, kg & $79.5 \pm 12.5$ & $83.3 \pm 13.4$ & $<0.001$ \\
\hline Mechanical ventilation & $681(72.7)$ & $356(77.1)$ & 0.08 \\
\hline Severe sepsis at baseline & $473(50.5)$ & $213(46.1)$ & 0.1 \\
\hline Non-operative admission diagnosis & $695(74.2)$ & $303(65.6)$ & 0.6 \\
\hline Operative admission diagnosis & $242(25.8)$ & $159(34.4)$ & 0.6 \\
\hline Severe acidemia $(\mathrm{pH}<7.2)$ & $361(38.5)$ & $132(28.6)$ & $<0.001$ \\
\hline Hemoglobin, g/l & $100.7 \pm 20.1$ & $97.8 \pm 18.8$ & 0.008 \\
\hline Platelet count, $\times 10^{9} / 1$ & $183.6 \pm 131$ & $184.3 \pm 133$ & 0.9 \\
\hline Sodium, mmol/l & $137.3 \pm 6.5$ & $138.3 \pm 6.7$ & 0.01 \\
\hline Chloride, $\mathrm{mmol} / \mathrm{l}$ & $105.0 \pm 7.4$ & $104.1 \pm 8.0$ & 0.05 \\
\hline CRRT dose during the first complete $24 \mathrm{~h}, \mathrm{ml} / \mathrm{kg} / \mathrm{h}$ & $27.5 \pm 8.2$ & $26.2 \pm 8.3$ & 0.01 \\
\hline Delivered/prescribed CRRT dose during the first complete $24 \mathrm{~h}, \%$ & $85.4 \pm 17.4$ & $81.9 \pm 19.8$ & 0.002 \\
\hline Duration first catheter/total CRRT time, $\%$ & $96.6 \pm 54.9$ & $94.5 \pm 84.2$ & 0.58 \\
\hline Death in ICU & $328(35.0)$ & $151(32.7)$ & 0.4 \\
\hline Death at day 90 & $410(43.8)$ & $208(45.1)$ & 0.6 \\
\hline
\end{tabular}

Variables are given as mean \pm SD or as numbers (percentage). APTT $=$ Activated partial thromboplastin time; INR $=$ international normalized ratio.

ride levels. CRRT was initiated $46.5 \mathrm{~h}$ after ICU admission in patients with femoral access and after $62.5 \mathrm{~h}$ in patients with non-femoral access $(p=0.02)$. The first femoral access was used during $96.6 \%$ of the total CRRT time and the first non-femoral access was used during $94.5 \%$ of the total CRRT time ( $\mathrm{p}=0.58$; table 1$)$. Overall, 410 (44\%) patients with a femoral dialysis catheter as first access had died at 90 days after randomization compared with 208 (45\%) non-femoral access patients (table 1).

Several factors were associated with a greater likelihood of the use of a femoral vein as the first site of catheter insertion on univariate logistic regression analysis. On multivariate logistic regression analysis, however, only body weight (OR 0.97 for each $\mathrm{kg}$ increase, 95\% CI 0.96-0.98), sodium levels (OR 0.93 for each mmol increase, 95\% CI 0.90-0.96) and chloride levels (OR 1.08 for each mmol increase, 95\% CI 1.05-1.11) were independently associated with choosing the femoral vein as the first site of access (table 2).

Femoral Access and Delivery of CRRT Dose

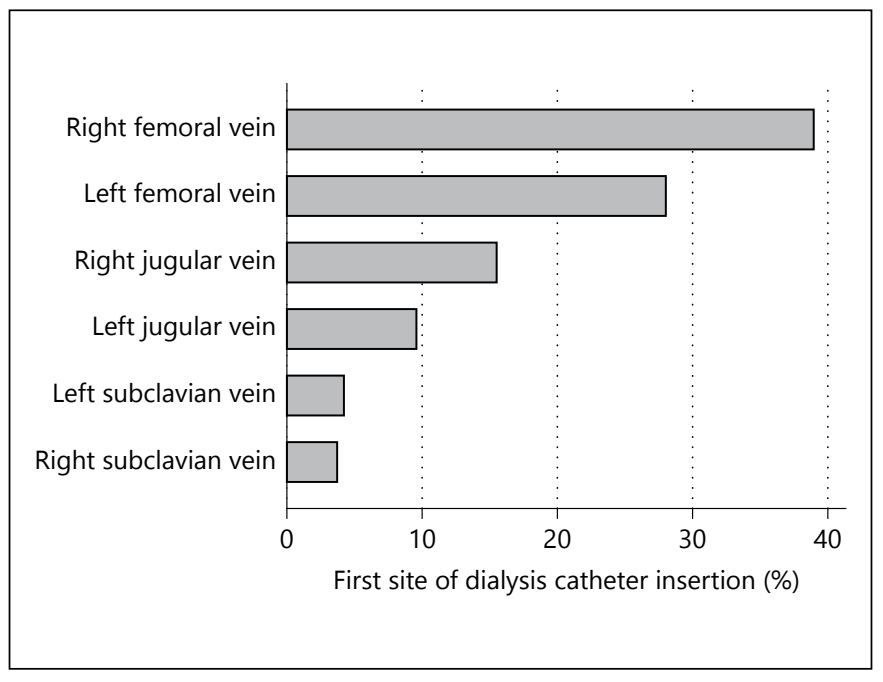

Fig. 1. Site of first temporary dialysis catheter insertion in the RENAL trial $(\mathrm{n}=1,399)$. 
Table 2. Univariate and multivariate logistic regression analysis of femoral vein as first site of catheter insertion

\begin{tabular}{|c|c|c|c|c|}
\hline \multirow[t]{2}{*}{ Variables } & \multicolumn{2}{|l|}{ Univariate analysis } & \multicolumn{2}{|c|}{ Multivariate analysis } \\
\hline & OR (95\% CI) & $\mathrm{p}$ values & OR (95\% CI) & $\mathrm{p}$ values ${ }^{\mathrm{a}}$ \\
\hline Body weight, kg & $0.977(0.97-0.99)$ & $<0.001$ & $0.972(0.96-0.98)$ & $<0.001$ \\
\hline Sodium, mmol/l & $0.979(0.96-1.00)$ & 0.01 & $0.928(0.90-0.96)$ & $<0.001$ \\
\hline Chloride, $\mathrm{mmol} / \mathrm{l}$ & $1.015(1.00-1.03)$ & 0.05 & $1.078(1.05-1.11)$ & $<0.001$ \\
\hline
\end{tabular}

a Adjusted for baseline characteristics with $\mathrm{p}<0.20$ in the univariate analyses. Only variables with $\mathrm{p}<0.05$ in the multivariate analysis are shown.

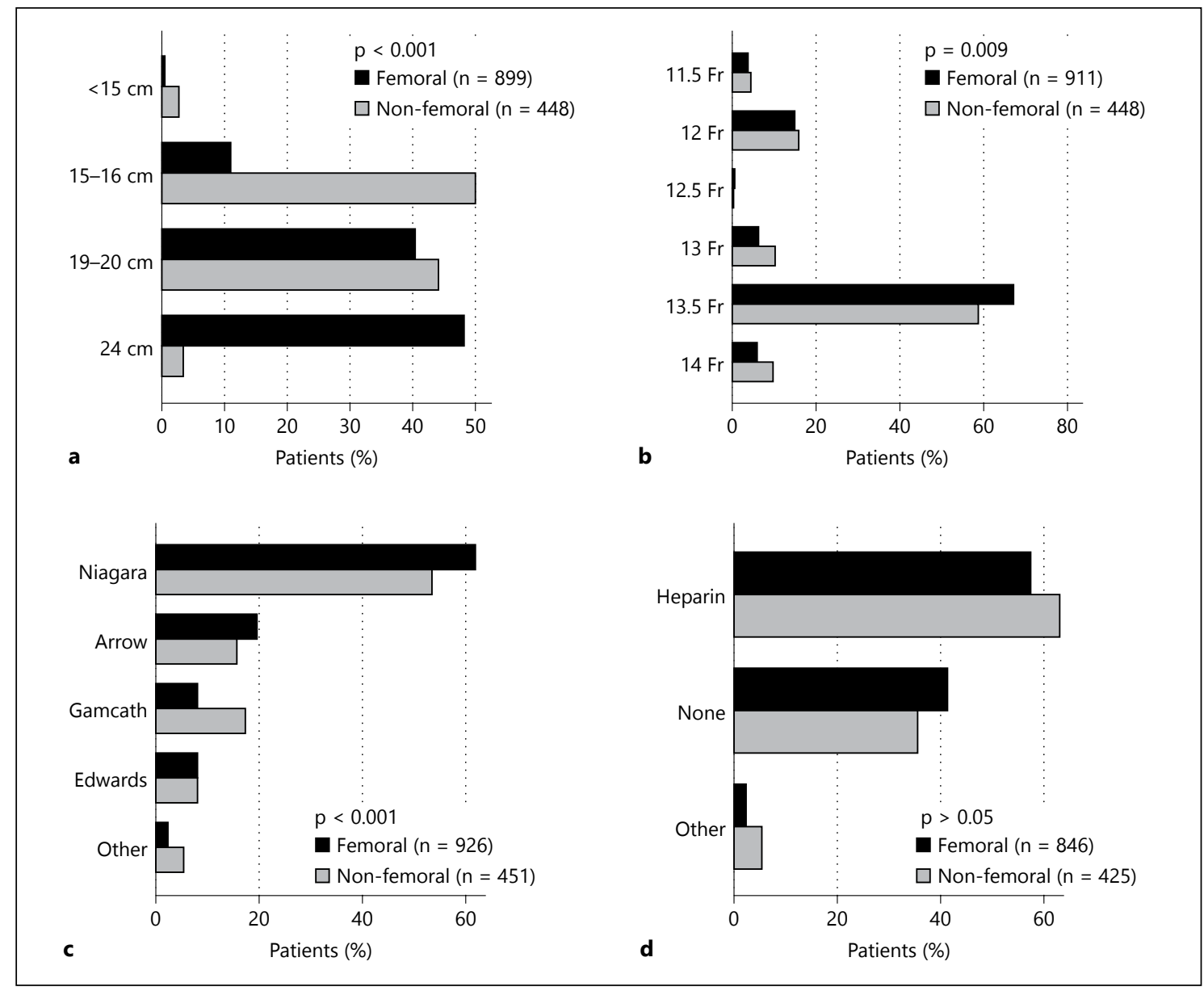

Fig. 2. Length (a), gauge (b) and brand (c) of dialysis catheter as well as circuit anticoagulation (d) during CRRT in the RENAL trial according to first site of catheter insertion.

\section{Catheter Characteristics and Circuit Anticoagulation}

Longer catheters were generally used for femoral access, whereas shorter catheters tended to be inserted in nonfemoral sites (fig. 2a). A 13.5-Fr catheter was most commonly chosen for both femoral (68\%) and non-femoral ac- cess (59\%; fig. 2b). Longer and greater gauge Niagara (Bard, Murray Hill, N.J., USA) catheters were mainly used in the femoral (62\%) and non-femoral (53\%) group (fig. 2c).

Information on circuit anticoagulation was available for $1,271(91 \%)$ patients. Heparin was used in 487 (58\%) 
Table 3. Multivariate linear regression showing the association with delivered CRRT dose (as \% of prescribed dose) during the first complete 24-hour period

\begin{tabular}{lcc}
\hline Variable & Coefficient, \pm SE & p values $^{\mathrm{a}}$ \\
\hline Femoral catheter (vs. non-femoral) & $-1.03 \pm 0.53$ & 0.05 \\
13.5 French catheter (vs. other gauges) & $4.20 \pm 1.90$ & 0.03 \\
20- or 24-cm catheter (vs. other lengths) & $1.88 \pm 1.68$ & 0.3 \\
Randomized to higher CRRT intensity & $-26.32 \pm 1.27$ & $<0.001$ \\
\hline
\end{tabular}

a Adjusted for age, sex, APACHE III score, body weight, oliguria, hyperkalemia, acidemia, oedema, urea, creatinine, catheter brand, CRRT machine use and mode of circuit anticoagulation. of 846 of patients in the femoral group and $269(63 \%)$ of 425 patients in the non-femoral group. CRRT was delivered without circuit anticoagulation in a high proportion of patients with femoral (41\%) and non-femoral (36\%) catheters $(\mathrm{p}=0.50$; fig. $2 \mathrm{~d})$.

\section{Factors Associated with Delivered CRRT Dose during} the First $24 \mathrm{~h}$

A total of 456 (48.7\%) patients with a femoral access were randomized to high intensity therapy $(40 \mathrm{ml} / \mathrm{kg} / \mathrm{h})$ compared to $236(51.1 \%)$ patients with a non-femoral catheter $(\mathrm{p}=0.40)$. On an average, CRRT intensity during the first complete $24 \mathrm{~h}$ was $27.5 \mathrm{ml} / \mathrm{kg} / \mathrm{h}$ in the femoral group and $26.2 \mathrm{ml} / \mathrm{kg} / \mathrm{h}$ in the non-femoral group $(\mathrm{p}=$ 0.01 ). On an average, 85.4 and $81.9 \%$ of the prescribed dose $(\mathrm{p}=0.002)$, respectively, was achieved during this period (table 1). On multivariate linear regression, adjusting for baseline characteristics, treatment group, catheter characteristics, CRRT machine and mode of circuit anticoagulation, the use of a femoral catheter was associated with a $1.03 \%$ lower CRRT dose $(\mathrm{p}=0.05)$ during the first $24 \mathrm{~h}$ than when a non-femoral catheter was used (table 3). In contrast, a $4.20 \%$ higher CRRT dose was achieved with 13.5 Fr gauge catheters than with other catheter gauges $(p=0.03)$. Finally, being randomized to higher intensity CRRT was independently associated with a $26.32 \%$ lower CRRT dose than being allocated to lower intensity CRRT.

\section{Discussion}

\section{Key Findings}

We analyzed the use of temporary dialysis catheters, the choice of insertion site, the catheter gauge and their impact on CRRT dose in critically ill patients enrolled in the RENAL trial. We found that the femoral vein was used as the first site of dialysis catheter insertion in two thirds of patients and was preferentially chosen in patients with lower body weight and in sicker patients. Using a femoral vein for vascular access had limited impact on the delivered early CRRT dose during the first $24 \mathrm{~h}$. In contrast, catheter gauge was a more important determinant of delivered CRRT dose during this time frame.

\section{Relationship to Previous Studies}

Previous studies investigating the impact of vascular access site on delivered RRT dose have reported inconsistent results. Hryszko et al. [12] found an independent 5 -fold higher risk of catheter dysfunction, defined as inability to maintain blood flow of at least $150 \mathrm{ml} / \mathrm{h}$, with femoral as compared to internal jugular catheters. In that study, however, $75 \%$ of patients were fully mobile, and treatment was with intermittent hemodialysis. Liangos et al. [13] explored dialysis performance during $>400$ intermittent hemodialysis sessions in 81 patients with acute renal failure. Use of a femoral access, as compared to a non-femoral access, was independently associated with lower urea reduction ratio with intermittent hemodialysis. Of note, however, the femoral catheters used in this study were short, measuring only 16.5 or $19.5 \mathrm{~cm}$. To optimize blood flow and CRRT dose and to avoid recirculation, it is important that the catheter tip is placed in a large vein (external iliac and close of inferior vena cava) or in the right atrium $[5,14,15]$. Therefore, it is recommended that $24-\mathrm{cm}$ catheters be used in the femoral position, and that $15-20 \mathrm{~cm}$ catheters be used for right jugular cannulations [16]. In 11 of the 12 centers involved in the French Cathedia study, 16-cm catheters were used in the jugular and $25-\mathrm{cm}$ catheters in the femoral position [17]. Similarly, 24-cm catheters were frequently used for femoral (48\%) and 15- to $16-\mathrm{cm}$ catheters were frequently used for jugular or subclavian access (50\%) in the RENAL study patients.

The Cathedia study showed a higher rate of catheterrelated infections with a femoral access in patients with 
body mass index $>28.4 \mathrm{~kg} / \mathrm{m}^{2}$ [17]. Furthermore, the increased catheter-related infections risk in heavier patients $(>90 \mathrm{~kg}$ ) with femoral dialysis catheters was recently confirmed in an observational study involving 458 patients [18]. It is therefore not surprising that RENAL study patients receiving right or left femoral catheterization [19] had a lower body weight than patients receiving non-femoral catheterization.

\section{Implications of Study Findings}

Our study suggest that clinicians perceive the femoral vein to be a useful potential first choice when rapid and safe access is needed and in patients with a multilumen central venous catheter in the right jugular vein [6]. Our results reflect this preference with sicker patients (more severe acidemia, higher APACHE III and cardiovascular Sequential Organ Failure Assessment score and faster initiation of CRRT after ICU admission) being more likely to have their first CRRT catheter inserted in the femoral vein. This, in turn, may reflect the fact that a central venous catheter is often inserted before a temporary dialysis catheter in hemodynamically unstable patients. Under such circumstances, the femoral route may be the only available option. Finally, we found that a femoral vein is preferred in patients with lower body weight. Although we did not assess body habitus, this finding may reflect the fact that femoral vein cannulation may be technically difficult and is therefore avoided in obese patients. In the univariate comparison, the delivered CRRT dose (as a percentage of the prescribed dose) was 3.5\% higher through a femoral access than through a non-femoral access (table 1). A femoral access was, however, associated with $1.03 \%$ lower CRRT delivery in the multivariate linear regression analysis. The fact that a higher proportion of patients with femoral access were cannulated with large-gauge $13.5 \mathrm{Fr}$ catheters as compared to patients with non-femoral access ( 67.5 vs. $59.2 \%, p=0.009$ ) likely explains the reversed result in the multivariate adjusted analysis. In fact, after adjusting for several potentially important confounders of the relationship between access site and dose, larger catheter gauge (13.5 Fr) was independently associated with delivery of a higher (4.2\%) CRRT dose whereas the ability to achieve the prescribed dose was, as could be expected, impaired in patients allocated to higher intensity CRRT. These findings need to interpreted in the light of the original RENAL trial results. In the RENAL trial, the high-intensity group received a $50 \%$ higher CRRT dose than the low-intensity group. Despite this difference in dose, 90-day mortality was similar in both the groups. Our results therefore suggest that insertion of 13.5 Fr gauge CRRT catheters in the femoral vein of very sick critically ill patients have no clinically important negative impact on early CRRT delivery. This is an important finding since femoral catheter insertion is a safer and easier approach for less experienced, junior doctors than cannulation of internal jugular or subclavian veins.

\section{Strengths and Limitations}

This study has important strengths. All study data, including highly detailed information on catheter characteristics, insertion site and CRRT dose were prospectively collected. In addition, the large study size enabled adjustment for numerous factors such as patient characteristics, catheter features and mode of anticoagulation, which may potentially affect CRRT delivery. With such adjustments, important practical observations emerged, which can be used to inform clinical practice.

This study has limitations. Choice of catheter insertion site and gauge were not randomized. However, our study provides the largest description of CRRT catheter characteristics, gauge and site of insertion to date and detailed information to adjust for many key early confounders. No information on catheter tip placement was available. However, since longer catheters were used in the femoral position and since non-femoral catheters were mainly inserted in the right jugular vein, it is likely that most of the catheters were positioned correctly. Our study does not provide information on the impact of insertion site or gauge on CRRT delivery beyond the first complete $24 \mathrm{~h}$. This was, however, our intention since determinants of CRRT dose unrelated to access, such as interruptions for investigations, operations and mortality would create a strong competing risk-related confounding effect, given that femoral catheters were inserted in sicker patients. By restricting the analysis to the first complete $24 \mathrm{~h}$ of CRRT, we aimed to diminish the impact of such confounders.

\section{Conclusions}

The femoral vein was used as the first site of dialysis catheter insertion in most patients within the RENAL trial, especially in lighter and sicker patients. Although such use of a femoral access was significantly associated with lower CRRT dose, the impact on dose was limited. However, using a larger gauge catheter was independently as-
Bellomo et al. 
sociated with the ability to deliver a higher CRRT dose during the first $24 \mathrm{~h}$. This information provides clinically relevant information to assist clinicians in their choice of preferred access site and catheter gauge for the delivery of CRRT in critically ill patients.

\section{Acknowledgments}

\section{Contributions}

Research idea and study design: R.B., A.C., M.G.; data analysis/interpretation: J.M., R.B., S.L.; statistical analysis: S.L.; supervision and mentorship: R.B.; drafting of manuscript: J.M., K.M.K., R.B. Each author contributed important intellectual content during manuscript revision and accepts accountability for the overall work by ensuring that questions pertaining to the accuracy or integrity of any portion of the work are appropriately investigated and resolved. R.B. and M.G. take responsibility that this study has been reported honestly, accurately and transparently; that no important aspects of the study have been omitted and that any discrepancies from the study as planned have been explained.

\section{Support and Financial Disclosure Declaration}

This study was supported by grants from the National Health and Medical Research Council (NHMRC) of Australia (grant 352550) and Health Research Council (HRC) of New Zealand (grant 06-357). The study sponsors had no role in study design; collection, analysis, and interpretation of data; writing the report; or the decision to submit the report for publication.

Professor A. Cass received funding from the Australian National Health and Medical Research Council and grant support from Roche and Gambro. Professor R. Bellomo received consulting fees as an advisor for Gambro; and consulted for Gambro, Biosite, Abbott and Philipps. Dr. M. Gallagher received honoraria/speaking fees from Roche. Dr. J. Mårtensson received travel support to present research results at scientific meetings from Gambro and funding from Karolinska Institutet. The remaining authors have not disclosed any potential conflicts of interest.

The George Institute has received research funding from Servier, Novartis, Eisai, Merck, Sharp \& Dohme, Pfizer Australia, Fresenius Kabi Deutschland GmbH and Sanofi Aventis.

The RENAL Replacement Therapy Study is a collaboration of the Australian and New Zealand Intensive Care Society Clinical Trials Group (ANZICS CTG) and the George Institute for International Health.

\section{References}

1 Joannidis M, Oudemans-van Straaten HM: Clinical review: patency of the circuit in continuous renal replacement therapy. Crit Care 2007;11:218.

2 Parienti JJ, Mégarbane B, Fischer MO, Lautrette A, Gazui N, Marin N, et al: Catheter dysfunction and dialysis performance according to vascular access among 736 critically ill adults requiring renal replacement therapy: a randomized controlled study. Crit Care Med 2010;38:1118-1125.

3 Beitland S, Sunde K, Moen H, Os I: Variability in uremic control during continuous venovenous hemodiafiltration in trauma patients. Crit Care Res Pract 2012;2012:869237.

4 Fealy N, Baldwin I, Bellomo R: The effect of circuit 'down-time' on uraemic control during continuous veno-venous haemofiltration. Crit Care Resusc 2002;4:266-270.

5 Morgan D, Ho K, Murray C, Davies H, Louw $\mathrm{J}$ : A randomized trial of catheters of different lengths to achieve right atrium versus superior vena cava placement for continuous renal replacement therapy. Am J Kidney Dis 2012; 60:272-279.

6 Dugué AE, Levesque SP, Fischer MO, Souweine B, Mira JP, Megarbane B, et al: Vascular access sites for acute renal replacement in intensive care units. Clin J Am Soc Nephrol 2012;7:70-77.
7 Kim IB, Fealy N, Baldwin I, Bellomo R: Insertion side, body position and circuit life during continuous renal replacement therapy with femoral vein access. Blood Purif 2011;31:42-46.

8 Lameire N, Kellum JA: Contrast-induced acute kidney injury and renal support for acute kidney injury: a KDIGO summary (part 2). Crit Care 2013;17:205.

9 Finfer S, Cass A, Gallagher M, Lee J, Su S, Bellomo R: The RENAL (randomised evaluation of normal vs. augmented level of replacement therapy) study: statistical analysis plan. Crit Care Resusc 2009; 11:58-66.

10 Bellomo R, Cass A, Cole L, Finfer S, Gallagher M, Goldsmith D, et al: Design and challenges of the randomized evaluation of normal versus augmented level replacement therapy (RENAL) trial: high-dose versus standarddose hemofiltration in acute renal failure. Blood Purif 2008;26:407-416.

11 Bellomo R, Cass A, Cole L, Finfer S, Gallagher $\mathrm{M}$, Lo S, et al: Intensity of continuous renalreplacement therapy in critically ill patients. N Engl J Med 2009;361:1627-1638.

12 Hryszko T, Brzosko S, Mazerska M, Malyszko J, Mysliwiec M: Risk factors of nontunneled noncuffed hemodialysis catheter malfunction. A prospective study. Nephron Clin Pract 2004;96:c43-c47.

13 Liangos O, Rao M, Ruthazer R, Balakrishnan VS, Modi G, Pereira BJ, et al: Factors associated with urea reduction ratio in acute renal failure. Artif Organs 2004;28:1076-1081.
14 Leblanc M, Fedak S, Mokris G, Paganini EP: Blood recirculation in temporary central catheters for acute hemodialysis. Clin Nephrol 1996;45:315-319.

15 Little MA, Conlon PJ, Walshe JJ: Access recirculation in temporary hemodialysis catheters as measured by the saline dilution technique. Am J Kidney Dis 2000;36:1135-1139.

16 Mrozek N, Lautrette A, Timsit JF, Souweine B: How to deal with dialysis catheters in the ICU setting. Ann Intensive Care 2012;2:48.

17 Parienti JJ, Thirion M, Mégarbane B, Souweine B, Ouchikhe A, Polito A, et al: Femoral vs jugular venous catheterization and risk of nosocomial events in adults requiring acute renal replacement therapy: a randomized controlled trial. JAMA 2008;299:24132422.

18 Chua HR, Schneider AG, Sherry NL, Lotfy N, Chan MJ, Galtieri J, et al: Initial and extended use of femoral versus nonfemoral double-lumen vascular catheters and catheter-related infection during continuous renal replacement therapy. Am J Kidney Dis 2014;64:909917.

19 Crosswell A, Brain MJ, Roodenburg O: Vascular access site influences circuit life in continuous renal replacement therapy. Crit Care Resusc 2014;16:127-130. 